\title{
HUMEDALES DE LA COSTA CENTRAL DEL PERÚ: ESTRUCTURA Y AMENAZAS DE SUS COMUNIDADES VEGETALES
}

\section{WETLANDS OF THE CENTRAL COAST OF PERU: STRUCTURE AND MENACES OF ITS VEGETAL COMMUNITIES}

\author{
Héctor Aponte Ubillús ${ }^{1}$ y Dámaso W. Ramírez Huaroto²
}

\begin{abstract}
Resumen
Los humedales de Lima son parte de un corredor biológico a lo largo de la costa desértica del Perú. A pesar de los múltiples estudios florísticos realizados en estos ambientes, poco se conoce acerca de estructura comunitaria. Con el objetivo de conocer la estructura de las comunidades vegetales se realizó un análisis cuantitativo por transectos y su posterior evaluación con métodos multivariados. Cuatro humedales fueron estudiados (Puerto Viejo, Cañete; Santa Rosa, Huaral; Paraíso, Chancay y Medio Mundo, Végeta). Se encontró que cada humedal presenta una estructura particular y compleja de sus comunidades vegetales, la cual guarda íntima relación con las actividades antrópicas de cada localidad. Se identificó a la ganadería y agricultura como una de sus principales amenazas. Se propone que la estructuración de las comunidades vegetales es un potencial bioindicador del estado de conservación a considerar dentro de los planes de manejo y monitoreo de estas áreas naturales.
\end{abstract}

Palabras clave: humedal, vegetación, comunidades, conservación, Lima

\begin{abstract}
Lima's wetlands are part of a biological corridor along the desert coast of Peru. Despite the large number of floristic studies conducted in these environments, little is known about the plant structure of their communities. In order to assess the structure of the plant communities a quantitative analysis using transects was conducted with further evaluation using multivariate methods. Four wetlands were chosen (Puerto Viejo, Cañete; Santa Rosa, Huaral; Paraíso, Chancay y Medio Mundo, Végueta). It was found out that each wetland had its own community structure, which is related to anthropic activities in each locality. Agriculture and livestock were identified as the main menaces in these communities. It is proposed that community plant structure is a potential bio indicator of the condition of the wetland and should be taken into consideration in management and monitoring plans of natural areas.
\end{abstract}

Key words: wetland, vegetation, communities, conservation, Lima

\section{Introducción.}

Los humedales de Lima son parte de un corredor biológico a lo largo de la costa desértica del Perú. La mayor parte de estos ambientes coexiste con poblaciones humanas, quienes hacen uso de los recursos que los humedales les proporcionan, por ejemplo Schoenoplectus americanus (Pers.) Volkart ex Schinz \& R. Kéller (conocido como «junco ») es utilizado para la fabricación de productos artesanales (León \& Young, 1996; León et al 1997). Otras actividades en estos ecosistemas son la pesca artesanal y la crianza de ganado vacuno y ovino. En ese sentido, la relación hombre-naturaleza tiene un lado productivo positivo. Sin embargo, esta relación también presenta un aspecto negativo, la extracción del junco en algunos humedales genera desechos que promueven la presencia de plagas (por ejemplo, la polilla y la quereza del junco) las cuales dañan diversas especies nativas (Sánchez, 2007; Aponte, 2009). Otros humedales reciben aguas servidas y desechos orgánicos de poblaciones humanas aledañas, afectando la calidad del agua en estos ambientes (Young, 1998).

Trabajos previos en Pantanos de Villa (Lima) han permitido identificar las cinco formaciones vegetales más importantes: Totoral, Vega de ciperáceas, Gramadal, Acuáticas, y Zona arbustiva (Young, 1998; Cano et al., 1993; Cano et al., 1998; Arana, 1998). Sin embargo, pocos han sido los esfuerzos por determinar la estructura de las comunidades vegetales de forma cuantitativa en estos ambientes (Ramírez et al., 2010). Siendo las especies vegetales y sus comunidades componentes importantes de este tipo de ecosistemas, el estudio y monitoreo de las misma nos permite tener una idea del estado actual de la diversidad de estos 
ambientes y de las actividades de gestión necesarios para su manejo y conservación a largo plazo.

El objetivo del presente trabajo fue analizar cuantitativamente la estructura de las comunidades vegetales e identificar las principales amenazas antrópicas.

\section{Área de estudio.}

Los humedales estudiados se ubican en la costa central del Perú, en el departamento de Lima, entre los $10^{\circ} 544^{`}$ “ y los $12^{\circ} 34$ S, desde el nivel del mar hasta altitudes no mayores de $25 \mathrm{~m}$ (Figura 1). Los humedales estudiados corresponden a:

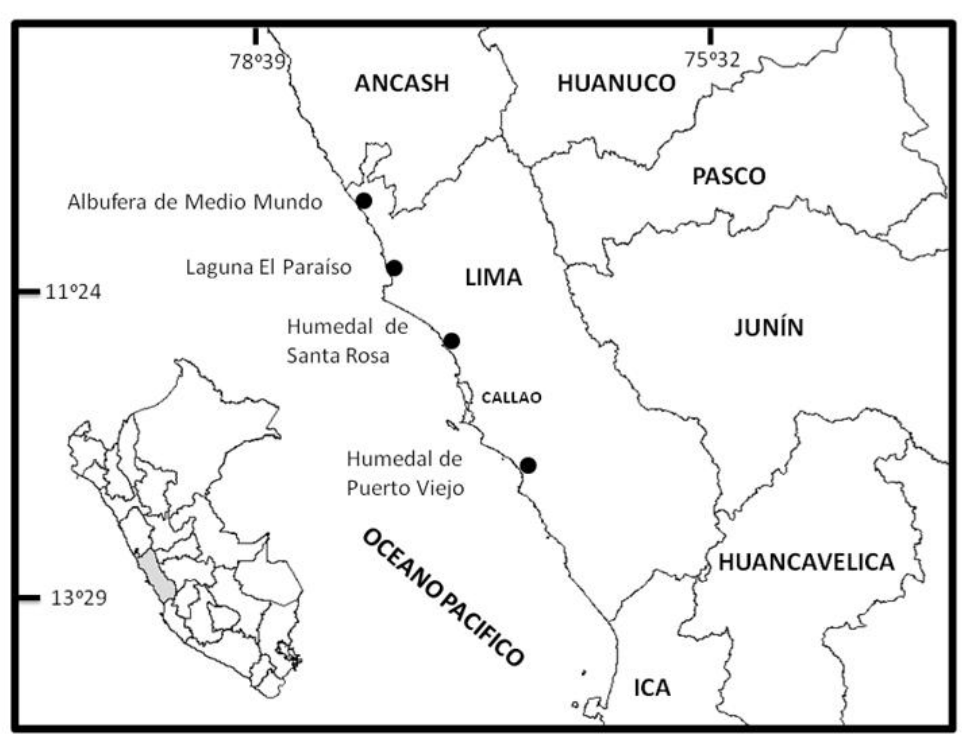

Figura 1. Mapa de la Costa Central del Perú. A la izquierda, el mapa del Perú y en gris el departamento de Lima. La ubicación de los cuatro humedales se encuentra señalada con un punto negro.

A)Los Humedales de Puerto Viejo, se localizan al sur de Lima, en la provincia de Cañete en el distrito de San Antonio de Mala, a la altura del Km 71 de la panamericana Sur (12³4's; 76² $\left.42^{\prime} \mathrm{O}\right)$ a 22 msnm. Este humedal ha sido formado por las afloraciones hídricas del acuífero de Mala, que están ubicadas paralelamente al océano. Esta localidad tiene aproximadamente 200 ha $y$ comprende varios cuerpos de agua zonas pantanosas y terrenos calcáreos (La Torre \& Aponte, 2009). En esta localidad hay zonas utilizadas para la ganadería ovina y vacuna, así como pequeñas áreas de extracción de junco (Schoenoplectus americanus) y totora balsa (Schoenoplectus californicus) (Aponte, observación personal). Parte de esta área es de propiedad privada. A pesar de ello cuenta con la categoría de Zona Reservada.

B)El Humedal de Santa Rosa se encuentra al norte del departamento de Lima, provincia de Huaral, distrito de Chancay, localidad de Santa Rosa con coordenadas $11^{\circ} 36^{\prime} \mathrm{S}$ y $77^{\circ} 15^{\prime} \mathrm{O}$; la altitud varía desde el nivel del mar hasta los $10 \mathrm{~m}$. Tiene un área aproximada de 32 ha, la cual comprende un gran cuerpo de agua en la zona central, una pequeña laguna al lado oeste y un canal principal que lo abastece de agua proveniente del río Chancay. Presenta un terreno plano a ligeramente ondulado y sus tierras son aptas para la producción de pastos y cultivos. El humedal se encuentra rodeado por varias fuentes de impacto antropogénico como la agricultura, ganadería, depósitos de desperdicios orgánicos y criaderos de porcinos. Gran parte de esta localidad tiene propietarios legales (quienes pertenecen a la localidad de Peralvillo y Salinas Alta) que utilizan la zona Sur y Este como campos de cultivo, entre los que predominan el cultivo de algodón (Gossypium barbadense), camote (Ipomea batatas), fresas (Fragaria sp.) y zapallo (Cucurbita pepo). En la zona oeste del humedal, hacia el límite con el Océano Pacifico, se presenta un reservorio de desperdicios y unos criaderos de porcinos. Hacía el lado este y norte está la zona urbana de Santa Rosa. La zona este del humedal es utilizada frecuentemente como área de pastoreo ovino y vacuno. Esta localidad tiene la categoría de Área de Conservación Municipal y se encuentra a cargo de la Municipalidad de Chancay.

C) La laguna El Paraíso se encuentra situada a unos $140 \mathrm{~km}$ al norte de la ciudad de Lima, en el distrito de Huacho, provincia de Chancay $\left(11^{\circ} 13^{\prime}-11^{\circ} 10^{\prime} S\right.$; $77^{\circ} 35-$ $\left.77^{\circ} 40^{\prime} O\right)$. Esta Laguna, formada en 1973 como consecuencia de filtraciones agrícolas de la irrigación Santa Rosa, tiene una longitud máxima de $8 \mathrm{~km}$, con un ancho que va desde los 0.1 y 2 $\mathrm{km}$, así como una profundidad máxima de 1,5 m (Riveros et al., 1983; Castro et al., 1990). Hacia el lado sur del humedal hay una zona en contacto con el mar, lo que hace que en momentos de marea alta la laguna se vea inundada por agua de mar. Esta localidad es una de las zonas de mayor extracción del junco de Lima y presenta pequeños grupos de ganado vacuno y ovino (Cruz, 2002). En esta localidad son frecuentes las actividades de extracción de junco (Schoenoplectus americanus) y totora (Typha domingensis).

D)La Albufera de Medio Mundo está situada entre los kilómetros 150 y 175 de la carretera 
Panamericana Norte, en el distrito de Végueta, provincia de Chancay (1056'S; $\left.77^{\circ} 41^{\prime} \mathrm{O}\right)$. Tiene aproximadamente un largo de $6.5 \mathrm{~km}$ y un ancho de 165 y 525 m, con un área total de 261.5 ha, de las cuales, 206 ha corresponden al espejo de agua y 55.5 ha a la parte pantanosa (Velit, 1974). La laguna se encuentra formada en su mayor parte por agua proveniente del mar. Como actividad productiva en este humedal se realiza la extracción de junco (Schoenoplectus americanus) y carrizo (Arundo donax) así como el ecoturismo. Por otro lado, hacia el lado este del humedal se encuentra una granja de crecimiento de pollos. Esta área cuenta con la categoría de Área de Conservación Regional (ACR).

\section{Métodos.}

1. Análisis de las Comunidades Vegetales

Con la finalidad de analizar las distintas comunidades vegetales de cada humedal, se realizó una evaluación por transectos. Se realizaron un total de 120 transectos (30 por localidad) a lo largo de todo el periodo de estudio (Enero - Junio, 2009), los cuales fueron ubicados sobre las diferentes formaciones vegetales encontradas en cada humedal. Cada formación vegetal fue identificada inicialmente basada en su fisonomía (Cano et al., 1998). En cada transecto se aplicó el método "Point Quadrat" con modificaciones (Ramírez et al., 2010). Este método permite caracterizar la vegetación mediante la cobertura y es adecuado para las comunidades vegetales donde no es fácil discriminar los individuos (pastizales y matorrales). Cada transecto tuvo una longitud de $10 \mathrm{~m}$. Se tomó un total de 50 puntos por transecto. En cada punto se registraron las especies que tocaron una varilla de 1.5 metros. Asimismo se anotó la cantidad de veces que cada especie tocaba la varilla a fin de obtener una idea de la cobertura en el punto. Si no se encontraba nada en ese punto se consideró vacío. La cantidad de puntos vacíos fue restada de los 50 puntos totales siendo utilizado como medida de cobertura (puntos llenosx100/puntos totales).

Los datos obtenidos de la evaluación de transectos fueron analizados mediante un análisis de correspondencia (AC). Asimismo se halló el índice de dominancia de Simpson (D) entre las especies, el cual varía desde 0 (equitabilidad) hasta 1 (dominancia completa de una especie). Estos análisis se realizaron mediante el software PAST 1.89 (Hammer et al., 2001).

\section{Resultados.}

Mediante el análisis de correspondencia, fueron reconocidas quince comunidades vegetales en los cuatro humedales muestreados, cada humedal presentó de tres a cinco comunidades (Figuras 2 y 3 ).
Asimismo el análisis de correspondencia mostro que cada humedal presenta una estructura particular de sus comunidades vegetales. Los datos obtenidos de riqueza, cobertura y dominacia por transectos se muestran en la Tabla 1.

En el Humedal de Puerto Viejo fueron encontradas tres comunidades (Figura 2a): una comunidad dominada por Sesuvium portulacastrum de escasa cobertura (40\%) y de dominancia alta $(D=1)$; otra comunidad de borde de laguna dominada por Paspalidium geminatum, encontrada solamente hacia el lado este del humedal de cobertura y dominancia altas $(100 \% \quad$ y $\quad \mathrm{D}=1$ respectivamente); y una comunidad de gramadal mixto dominado por Distichlis spicata acompañados de parches de Sarcocornia neei y Schoenoplectus americanus, esta comunidad ocupa la mayor extensión del área estudiada con zonas de $100 \%$ de cobertura y con dominancia variable entre los transectos $(D=0.36-1)$.

Para el Humedal de Santa Rosa se encontraron igualmente tres comunidades (Figura 2b): Una comunidad de acuáticas flotantes dominada por Pistia stratiotes, que ocupa la mayor cantidad de los cuerpos de agua con cobertura entre 66\% y $100 \%$ y dominancia alta $(\mathrm{D}=0.87-1)$; un totoral dominado por Typha domingensis con acuáticas asociadas como Eichhornia crassipes y Enhydra sessilifolia, esta comunidad presenta dominancia media $(D=0.5-0.79)$ y coberturas que alcanzan hasta el $100 \%$. Finalmente una comunidad de vega mixta que corresponde a una comunidad de porte bajo y posee gran cantidad de especies de ciperáceas, especies de gramadal, juncal e inclusive especies oportunista. Esta última comunidad se caracteriza por tener zonas de dominancia baja $(\mathrm{D}=0.26$ para el transecto 1 y $\mathrm{D}=0.27$ para los transectos 15 y 25) y gran cantidad de taxa en los transectos.

En el Humedal de Paraíso, cinco comunidades fueron identificadas (Figura 3a): Una comunidad de anfibias de borde de laguna dominada por Bolboschoenus maritimus con Chenopodium petiolare, muy frecuente hacia el lado norte del humedal con dominancia y coberturas de $\mathrm{D}=0.62 \mathrm{y}$ 92\% respectivamente; una comunidad de gramadal dominado por Distichlis spicata y Sporobulus virginicus caracterizado por tener algunas zonas de cobertura baja (hasta 54\%) y por tener dominancia intermedia ( $\mathrm{D}=0.5-0.90)$. Esta última comunidad muchas veces se superpone a una comunidad dominada por Sarcocornia neei que forma grandes extensiones de parches entre el gramadal y juncal hacia el lado sur del humedal, caracterizada por tener dominancia intermedia $(\mathrm{D}=0.27-0.59)$ y gran cobertura (desde $80 \%$ hasta 100\%). Se identifica también una comunidad de anfibias de borde de laguna dominada por Bacopa monnieri, Eleocharis geniculata y Triglochin striatum muy frecuente en la laguna sur del humedal, caracterizada por tener baja 


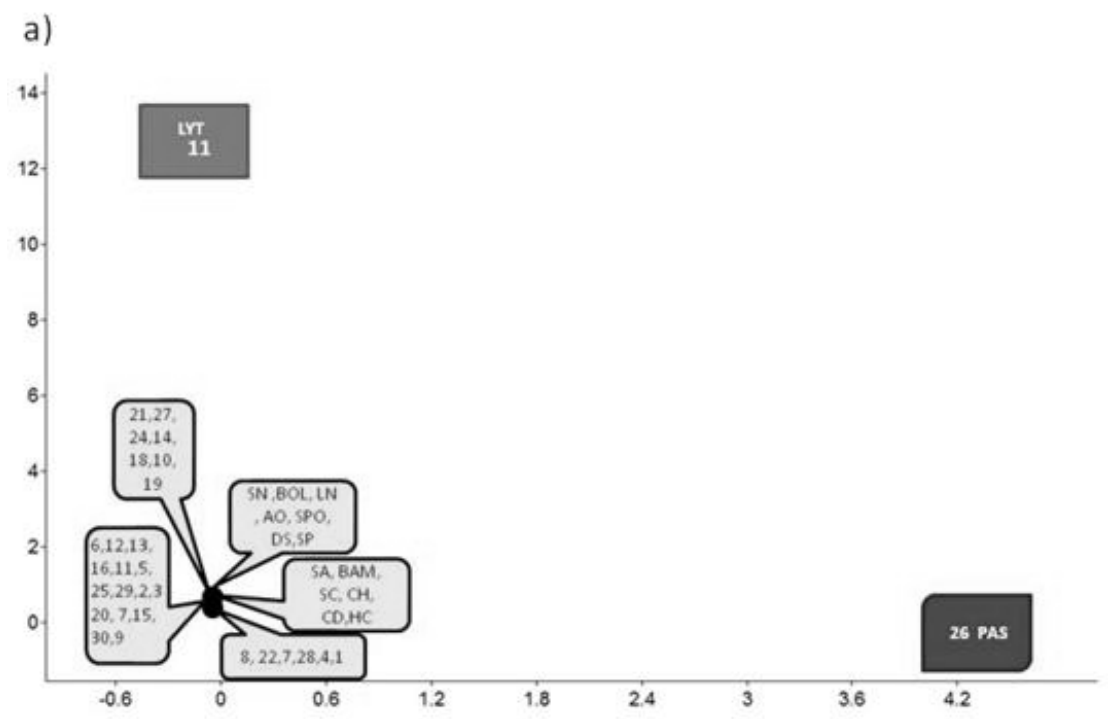

b)

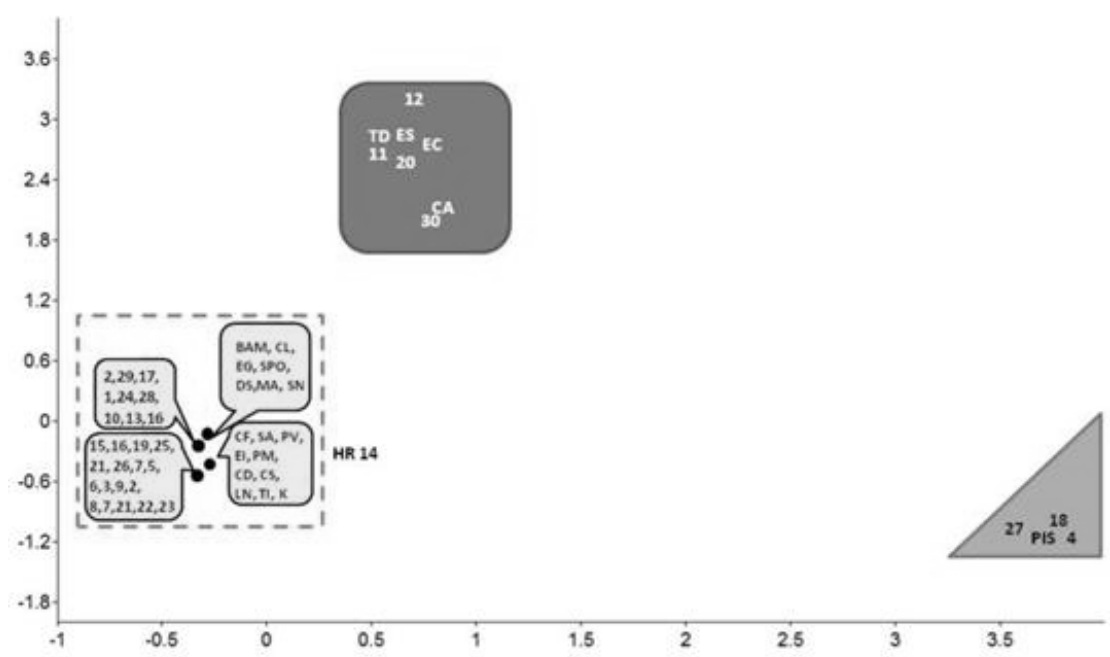

Figura 2. Resultados del Análisis de Correspondencia (AC) de las comunidades vegetales para los humedales de Puerto Viejo a) y Santa Rosa b). Los datos corresponden a los ejes 1 y 2 del análisis por ser los que incluyen la mayor variabilidad. Se muestran enumerados los transectos (1-30) y las especies características de las comunidades. PAS=Paspalidium geminatum; EC=Eichhornia crassipes; ES=Enydra sessilifolia; $\mathrm{TD}=$ Typha domingensis; $\mathrm{PS}=$ Pistia stratiotes; $\mathrm{BAM=Baccopa} \mathrm{monnieri;} \mathrm{SA}=$ Schoenoplectus americanus; $\mathrm{CD}=$ Cynodon dactylon; $\mathrm{DS}=$ Distichlis spicata; $\mathrm{CA}=$ Cyperus articulatus; $\mathrm{SN}=$ Sarcocornia nei; $\mathrm{PV}=$ Paspalum vaginatum; $\mathrm{Cl}=$ Cyperus laevigatus; $\mathrm{EG}=$ Eleocharis geniculata; $\mathrm{EI}=$ Eleocharis interstincta $\mathrm{HR}=$ Hydrocotyle ranunculoides; $\mathrm{LN}=$ Lippia nodiflora; $\mathrm{PM=Plantago}$ major; $\mathrm{CF}=$ Conmelinna fasciculata; $\mathrm{CS}=$ Colocassia esculenta; $\mathrm{TI}=$ Tessaria integrifolia; $\mathrm{SPO}=$ Sporobolus virginicus; $\mathrm{CH}=$ Chenopodium petiolare; $L Y T=$ Sessuvium portulacastrum.

dominancia y gran cobertura $(D=0.3$ y cobertura de 98\% para el transecto 19). Finalmente un comunidad de juncal que tiene en sus límites zonas de crecimiento de Typha domingensis y Schoenoplectus californicus, caracterizado por tener algunas zonas de cobertura alta (90\%-100\%) y una dominancia variable $(\mathrm{D}=0.38-1)$. A Cyperus laevigatus no se le asignó una comunidad característica al encontrársele en varias comunidades.
Los transectos 25, 3 y 18 representan zonas intermedias entre las comunidades identificadas en el área.

Por último, para el Humedal de Medio Mundo cuatro comunidades fueron identificadas (figura $3 b$ ): Una comunidad de anfibias de borde de laguna, dominada por Cyperus laevigatus y Chenopodium petiolare, con coberturas que varían entre 70\% y $80 \%$ 


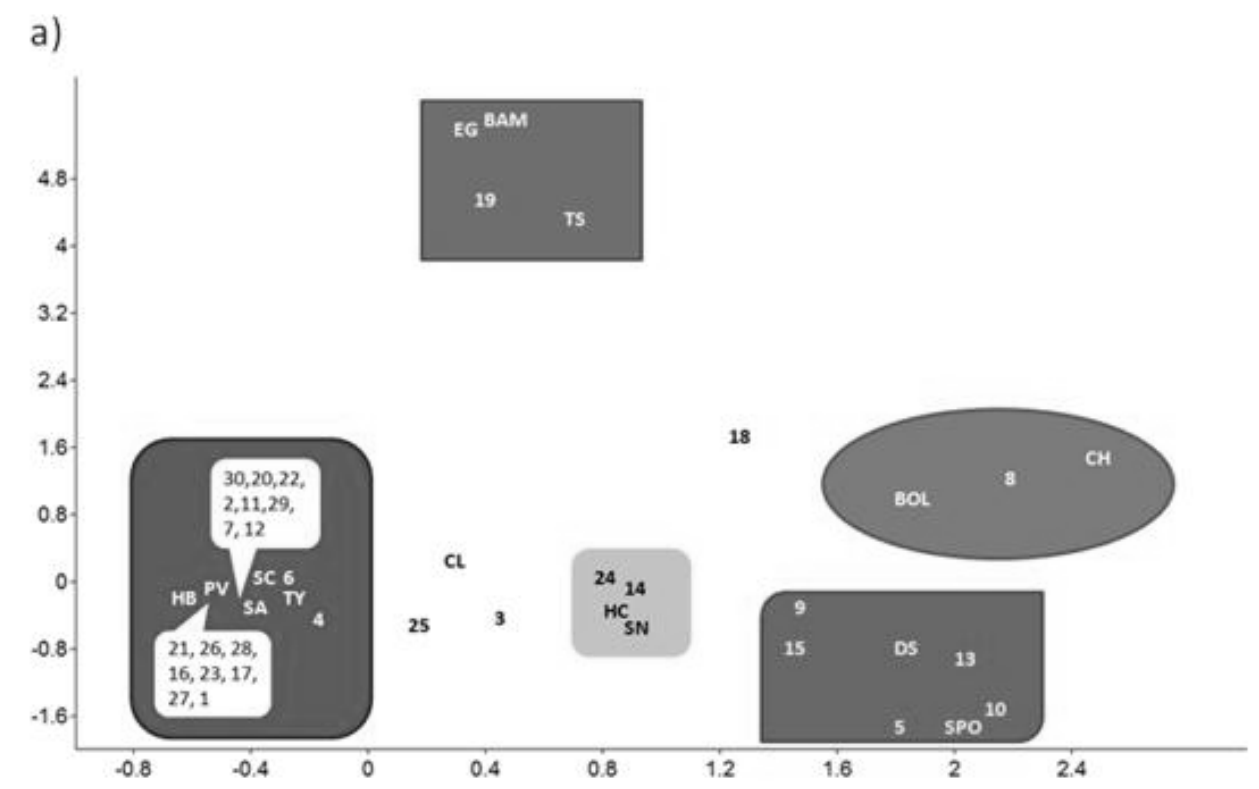

b)

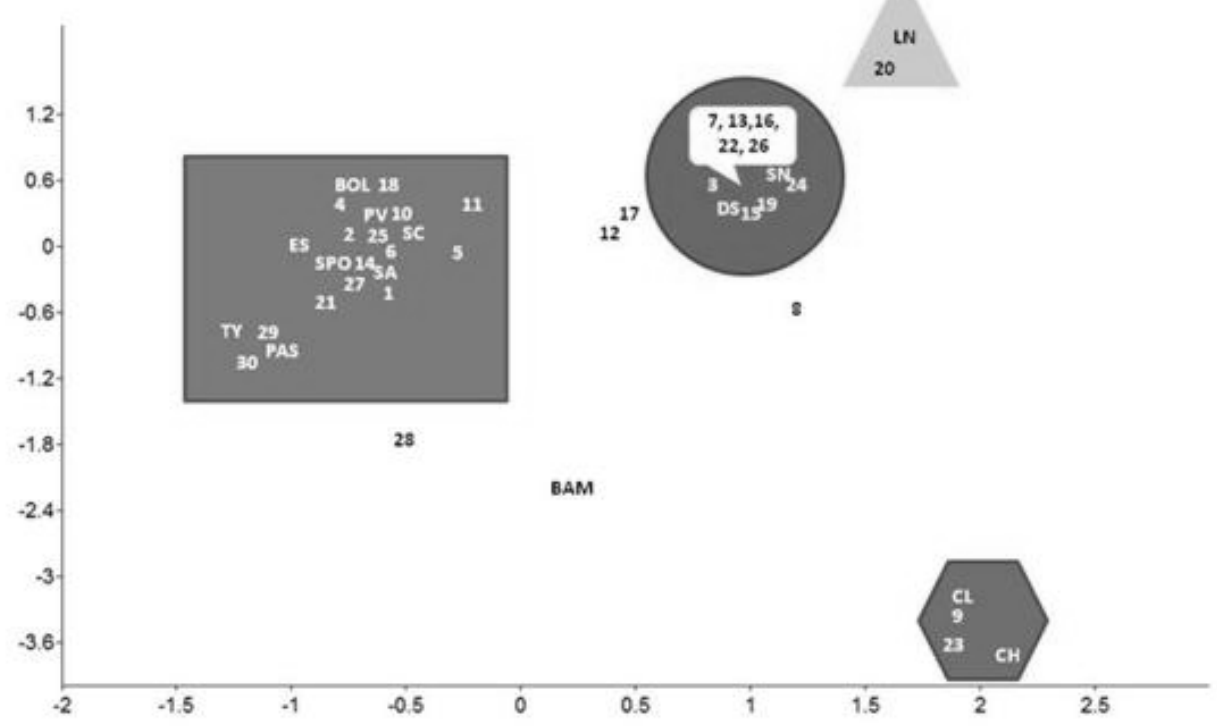

Figura 3. Resultados del Análisis de Correspondencia (AC) para los humedales de Paraíso a) y Medio Mundo b). Los datos corresponden a los ejes 1 y 2 del análisis por ser los que incluyen la mayor variabilidad. Se muestran enumerados los transectos (1-30) y las especies características de las comunidades. $\mathrm{BOL}=$ Bolboschoenus maritimus; ES=Enydra sessilifolia; TY=Typha domingensis; $\mathrm{BAM=Baccopa} \mathrm{monnieri;}$ $\mathrm{SA}=$ Schoenoplectus americanus; $\mathrm{SPO}=$ Sporobolus virginicus; $\mathrm{DS}=$ Distichlis spicata; $\mathrm{SN}=$ Sarcocornia neei; $\mathrm{PV}=$ Paspalum vaginatum; $\mathrm{Cl}=$ Cyperus laevigatus; $\mathrm{CH}=$ Chenopodium petiolare; $\mathrm{EG}=$ Eleocharis geniculata; $\mathrm{TS}=$ Triglochin striatum; HR=Hydrocotyle bonariensis; HC=Heliotropium curasavicum; LN=Lippia nodiflora; PAS=Paspalidium geminatum.

y de dominancia variable $(\mathrm{D}=0.54-0.82)$; una comunidad de gramadal dominada por Distichlis spicata y Sarcocornia neei con zonas de cobertura media (desde 58\% hasta 100\%) y de dominancia media y alta $(\mathrm{D}=0.52-1)$; una comunidad dominada por Lippia nodiflora, que se encuentra muy cerca al 
gramadal del lado este del humedal, con dominancia intermedia (0.59) y cobertura alta (100\%); y una comunidad de juncal que tiene como especie dominante a Schoenoplectus americanus y con especies de borde de laguna como Typha domingensis, Schoenoplectus californicus y Bolboschoenus maritimus, esta comunidad presentó zonas de cobertura hasta $100 \%$ y de dominancia media y alta ( $D=0.36-1)$. A Bacopa monnieri no se le agrupo en una comunidad propia, al haberse encontrado en varias de las comunidades determinadas. Asimismo los transectos 8, 12, 17, y 28 representan zonas intermedias entre las comunidades.

\section{Discusión.}

a) Estructura de las Comunidades Vegetales como indicador del impacto humano

Las comunidades vegetales obtenidas por el análisis cuantitativo son el reflejo de las actividades antrópicas en las formaciones vegetales originalmente presentes en cada humedal. La variación de su estructura es un indicador de las diferentes actividades antropicas que se practican en estos ecosistemas. Se han colectados datos de zonas con muy poca cobertura y baja riqueza de especies (en especial, a nivel del gramadal mixto), las cuales, están siendo afectadas por las actividades ganaderas y quemas. Asimismo, la extensión de los campos de cultivo y actividad ganadera, dan origen a comunidades que se caracterizan por una mezcla de especies nativas e introducidas, así también causa la reducción y alteración de formaciones vegetales como la vega de ciperáceas y el gramadal en los humedales (Ramírez et al, 2010). Esto da origen a comunidades tales como el gramadal mixto y la vega mixta encontradas en Puerto Viejo y Santa Rosa respectivamente. Estas actividades antrópicas pueden afectar no solamente las comunidades vegetales, sino también las comunidades de aves e insectos que utilizan los hábitats de los humedales (Pautrat \& Salcedo, 1998; Duarez, 1998). En las localidades donde las actividades ganaderas han dejado aún zonas intactas (como Paraíso y Medio Mundo) ha sido posible encontrar una mayor cantidad de comunidades, encontrando estructuras que guardan una mayor semejanza con las formaciones vegetales identificadas en estudios previos.

El aumento de las poblaciones de especies acuáticas como Pistia stratiotes, Eichhornia crassipes, y Lemna gibba, son posibles consecuencia de un exceso de nutrientes que llegan al humedal por actividades agrícolas y ganaderas lo que puede estar ocurriendo en el Humedal Santa Rosa. Considerando que muchas lagunas costeras del Perú son eutróficas (Aponte, 2007), sería importante poder seguir la población y la cantidad de nutrientes al mismo tiempo y analizar la correlación de este crecimiento poblacional con la cantidad de nutrientes en las lagunas.

Los resultados del presente estudio demuestran el uso potencial de la estructura y abundancia de las comunidades vegetales como un bioindicador de las actividades antrópicas, el cual debe ser considerado para futuros planes de monitoreo y manejo de los humedales estudiados.

Algunos trabajos previos en Pantanos de Villa (Young, 1998; Cano et al. 1993; Cano et al. 1998), mostraron la composición y características cualitativas de las formaciones vegetales en estos ecosistemas. El presente estudio permite conocer la estructura compleja que estas formaciones vegetales esconden, conociendo con mayor detalle la composición florística en estas comunidades, así como la dominancia entre las especies. Este resultado nos ayuda a comprender la necesidad de realizar estudios a nivel cuantitativo, que permitan establecer $\mathrm{y}$ caracterizar las comunidades vegetales, ya que con ello se lograra obtener mayor cantidad de información, que puede ser utilizada para la gestión de estos ecosistemas frágiles.

b) Importancia de la Gestión en los Humedales de la Costa Central

Considerando las amenazas identificadas para las comunidades vegetales, y haciendo una revisión de las actividades de gestión que se llevan a cabo en las localidades estudiadas, se observó que el establecimiento sin control de zonas ganaderas y agrícolas es una de las actividades que afecta evidentemente a las comunidades vegetales y a todo el humedal como ecosistema. El control de estas actividades debe ir de la mano con la protección gubernamental de estos ambientes. En las cuatro localidades estudiadas este proceso ya ha empezado con la elaboración de expedientes técnicos y la categorización de las áreas. Asimismo, este proceso requiere de actividades particulares en cada humedal, ya que, en algunos casos como en los Humedales de Santa Rosa y la Laguna El Paraíso, hay problemas de posesión de tierras (Cruz, 2002; Moya, 2005; Ramírez et al., 2010) que deberían ser aclarados prontamente. Por otro lado, varias ONG están apoyando para que se logre un acuerdo con la población y de esta forma se proteja correctamente estos ambientes tan importantes para el planeta.

Las áreas de estudio no son propicias para realizar actividades agrícolas ya que por su fisiografía sufren inundaciones periódicas que afectan los cultivos (Aponte \& Ramírez, observación personal). Las actividades agrícolas podrían ser reemplazadas por otras actividades económicas más rentables, como el uso gestionado del "junco" (como fuente de materia prima para construir cestos artesanales) y de Eichhornia crassipes (que por su biomasa podría ser utilizado de forma controlada como forraje) (Dada, 
2002). Estas actividades alternativas necesitan de un proceso de capacitación y concientización de la población y representan actividades de gestión a largo plazo, ya que si se implementa el uso del junco y/o totora se debe plantear programas de parcelización y calendarización (Sánchez, 2007; Aponte, 2009) y un manejo adecuado de los desechos que resulten de la extracción /producción.

En el caso puntual de las Albuferas de Medio Mundo, son necesarios estudios de evaluación de los efectos de la planta de crecimiento de pollos ubicado en la zona este y los impactos a nivel sonoro, visual y de contaminación química (Gonzales, 2007).

\section{Conclusión.}

El presente estudio muestra que las comunidades vegetales de cada humedal, esconden una estructura compleja y particular, la cual guarda una intima relación con las actividades antrópicas que se realizan en cada área estudiada. La principal amenaza identificada es la expansión agrícola y ganadera, la cual afecta a estas comunidades y al humedal como ecosistema, para lo cual se proponen medidas a implementar en la gestión de los humedales con el fin de conservar estos ecosistemas frágiles.

\section{Agradecimientos.}

A lo largo del estudio, se contó con la asesoría de diferentes actores de la gestión de los humedales entre los cuales destacan científicos del Museo de Historia Natural, principalmente del profesor Asunción Cano de quien quedamos profundamente agradecidos. Asimismo quisiéramos agradecer a Ricardo Jiménez Gerente de Ecología de la ONG Terra Nuova, Rosario Alcántara Bióloga encargada de la Gerencia de Recursos Naturales y Gestión del medio Ambiente del Gobierno Regional de Lima y a Federico Rizopatrón director del Centro Neotropical de Entrenamiento en Humedales quienes nos ayudaron en la búsqueda de información acerca de los humedales estudiados. Agradecemos a Marcelino Riveros, Kathia Tarqui, Randy Rosales, Susy Castillo, Huber Trinidad, Paúl Gonzales, Fresia Mejía y Elluz Huamán, por su valiosísimo apoyo en la etapa de campo del presente estudio. Finalmente, agradecemos a los revisores del presente artículo por sus valiosos comentarios y sugerencias.

\section{Literatura citada.}

Aponte H. 2007. Respuesta de Schoenoplectus americanus (Pers.) Vol. ex Sch. \& R. Séll. (Cyperaceae) “junco” a diferentes concentraciones de nutrientes. Tesis para optar al título profesional de Biólogo con mención en Botánica. Universidad Nacional Mayor de San Marcos, Facultad de Ciencias Biológicas.

Aponte H. 2009. El junco. Clasificación, Biología y gestión. Revista Científica 6(1), 2009. Universidad Científica del Sur. ISSN:1997-700X
Arana C. 1998. Relaciones fitogeográficas de la flora vascular de los pantanos de Villa. En : Los Pantanos de Villa: Biología y Conservación. A. Cano y K. Young (editores). Universidad Nacional Mayor de San Marcos (UNMSM). Serie de Divulgación Nº11. pp. 163-180.

Cano A., La Torre M.I., León B., Young K., Roque J. \& Arakaki M. 1998. Estudio comparativo de la Flora vascular de los Principales Humedales de las Zona Costera del Departamento de Lima, Perú. En: Los Pantanos de Villa: Biología y Conservación. A. Cano y K. Young (editores). Universidad Nacional Mayor de San Marcos (UNMSM). Serie de Divulgación $N^{\circ} 11$. pp. $181-190$

Cano A., León B. \& Young K.R. 1993. Plantas Vasculares de Los Pantanos de Villa. Pp. 177 - 207. En Kahn, F., B. León \& K. R. Young (compiladores). Las Plantas Vasculares en las Aguas Continentales del Perú. Instituto Francés de estudios Andinos (IFEA), tomo 75. Lima.

Castro G., Ortiz E. \& Bertochi L. 1990. Importancia Biológica y Conservación de la Laguna el Paraíso, Boletín de Lima 71:47-55. Lima.

Cruz Z. 2002. Evaluación del Estado de Conservación de las Lagunas "El Paraíso", Provincia de Huaura, Departamento de Lima, Perú. Tesis para optar por el título de Ingeniero Forestal.

Dada S. 2002. The utilization of water hyacinth (Eichhornia crassipes) by west african dwarf (wad) growing goats. Afr. J. Biomed. Res. (2002): Vol 4; 147 - 149.

Duarez J. 1998. Composición y riqueza de arañas (Arachnida: Araneae) en los Pantanos de Villa. En : Los Pantanos de Villa: Biología y Conservación. A. Cano y K. Young (editores). Universidad Nacional Mayor de San Marcos (UNMSM). Serie de Divulgación $N^{\circ} 11$. pp. $105-113$.

Gonzales J. 2007. Análisis preliminar del impacto ambiental de la empresa avícola Redondos en los Humedales de Paraíso y en el ACR Albúferas de Medio Mundo. Procomhcc - Terra Nuova SPDE. En: Corredor Biológico de la Costa Central del Perú - Base de Datos. Tomo VII.

Hammer O., Harper D.A.T. \& Ryan P.D. 2001. PAST: Paleontological Statistics Software package for of education and data analysis. Paleontologia Electrónica 4(1)9pp.

La Torre M.I. \& Aponte H. 2009 Flora Vascular y vegetación del Humedal de Puerto Viejo. Revista Peruana de Biología 16(2):215-217(Diciembre 2009)

León B. \& Young K. 1996. Aquatic plants of Perú: diversity, distribution and conservation. Biodiversity and Conservation 5 :1169-1190.

León B., Young K. \& Cano A. 1997. Fitogeografía y Conservación de la Costa Central del Perú. Estudios Sobre Diversidad y Ecología de plantas. Memorias del II Congreso Ecuatoriano de Botánica realizado en la Pontificia Universidad católica de Ecuador, Quito. 16-20 Oct. 1995. Pontificia Universidad Católica de Ecuador. Pp. 129-142.

Moya N. 2005. Humedal “El Paraíso”. Instituto de Promoción y Desarrollo Sostenible (INDESPE). En: Informe técnico de las Albúferas de Paraíso (Huaura Perú). Julio 2006. Iniciativa por la Conservación de la Costa Peruana. Guillermo Reaño y Alejandro Tello (editores). 
Pautrat L. \& Salcedo J.C. 1998. Evaluación de la avifauna de los Pantanos de Villa, Lima. En : Los Pantanos de Villa: Biología y Conservación. A. Cano y K. Young (editores). Universidad Nacional Mayor de San Marcos (UNMSM). Serie de Divulgación N¹1. pp. 85 -95.

Ramírez D., Aponte H. \& Cano A. 2010. Flora Vascular y Vegetación del Humedal De Santa Rosa (Chancay, Lima). Revista Peruana de Biología, 17(1): 105- 110 (Abril 2010).

Riveros J., Cayo M. \& Nuñez S. 1983. Censos de aves de importancia cigenética en las albúferas de Playa ChicaHuacho. Zonas áridas 3:75-81.

Sánchez R. 2007. Informe técnico del aprovechamiento del junco en los Humedales de la Costa central del Perú. En:
Corredor Biológico de la Costa Central del Perú - Base de Datos. Tomo I. Terra Nuova.

Velit E. 1974. Contribución al Conocimiento de las Relaciones tróficas de los peces de la Albúfera de "Medio Mundo" (costa central del Perú). Tesis de Bachiller en Ciencias Biológicas. Universidad Nacional Mayor de San Marcos. Lima.

Young K. 1998. El Ecosistema. En Los Pantanos de Villa: Biología y Conservación. A. Cano y K. Young (editores). Universidad Nacional Mayor de San Marcos. Serie de Divulgación №11. pp. 3-20. 
Tabla 1. Datos obtenidos a partir del análisis de transectos en las cuatro localidades evaluadas. Se muestra la cantidad de taxa (T) el número de toques(X), la dominancia (D) y la cobertura (C) por transecto.

\begin{tabular}{|c|c|c|c|c|c|c|c|c|c|c|c|c|c|c|c|c|c|c|c|c|c|c|c|c|c|c|c|c|c|c|}
\hline & 1 & 2 & 3 & 4 & 5 & 6 & 7 & 8 & 9 & 10 & 11 & 12 & 13 & 14 & 15 & 16 & 17 & 18 & 19 & 20 & 21 & 22 & 23 & 24 & 25 & 26 & 27 & 28 & 29 & 30 \\
\hline \multicolumn{31}{|c|}{ PUERTO VIEJO } \\
\hline $\mathrm{T}$ & 5 & 4 & 1 & 4 & 2 & 3 & 2 & 2 & 1 & 2 & 3 & 2 & 3 & 2 & 1 & 5 & 1 & 2 & 3 & 3 & 2 & 5 & 4 & 3 & 3 & 1 & 2 & 2 & 2 & 3 \\
\hline $\mathrm{X}$ & 83 & 169 & 65 & 153 & 152 & 159 & 106 & 159 & 133 & 146 & 21 & 85 & 161 & 164 & 180 & 120 & 77 & 109 & 144 & 215 & 60 & 205 & 133 & 97 & 145 & 212 & 133 & 188 & 137 & 141 \\
\hline $\mathrm{D}$ & 0.58 & 0.55 & 1 & 0.41 & 0.84 & 0.35 & 0.59 & 0.83 & 1 & 0.51 & 1 & 0.85 & 0.68 & 0.5 & 1 & 0.36 & 1 & 0.5 & 0.78 & 0.55 & 0.94 & 0.55 & 0.68 & 0.5 & 0.49 & 1 & 0.73 & 0.56 & 0.64 & 0.4 \\
\hline $\mathrm{C}$ & 80 & 100 & 70 & 100 & 100 & 100 & 100 & 100 & 90 & 100 & 40 & 80 & 100 & 100 & 100 & 100 & 100 & 90 & 100 & 100 & 80 & 100 & 90 & 100 & 100 & 100 & 100 & 100 & 100 & 100 \\
\hline \multicolumn{31}{|c|}{ SANTA ROSA } \\
\hline $\mathrm{T}$ & 5 & 3 & 1 & 1 & 2 & 4 & 8 & 9 & 6 & 9 & 4 & 2 & 1 & 1 & 4 & 3 & 5 & 2 & 3 & 3 & 4 & 6 & 4 & 4 & 5 & 3 & 2 & 6 & 5 & 5 \\
\hline $\mathrm{X}$ & 129 & 135 & 148 & 33 & 190 & 189 & 145 & 151 & 136 & 118 & 89 & 133 & 53 & 34 & 128 & 123 & 66 & 76 & 100 & 88 & 122 & 283 & 150 & 78 & 165 & 355 & 127 & 118 & 147 & 85 \\
\hline $\mathrm{D}$ & 0.26 & 0.9 & 1 & 1 & 0.67 & 0.44 & 0.42 & 0.62 & 0.37 & 0.56 & 0.61 & 0.53 & 1 & 1 & 0.27 & 0.43 & 0.63 & 0.97 & 0.54 & 0.79 & 0.66 & 0.37 & 0.9 & 0.52 & 0.27 & 0.66 & 0.87 & 0.37 & 0.51 & 0.5 \\
\hline C & 98 & 96 & 100 & 70 & 100 & 100 & 100 & 100 & 100 & 98 & 100 & 100 & 64 & 100 & 100 & 100 & 70 & 98 & 94 & 96 & 100 & 100 & 100 & 0.84 & 100 & 100 & 100 & 100 & 100 & 0.76 \\
\hline \multicolumn{31}{|c|}{ PARAÍSO } \\
\hline $\mathrm{X}$ & 3 & 3 & 4 & 5 & 2 & 4 & 3 & 2 & 3 & 2 & 3 & 3 & 2 & 5 & 2 & 3 & 3 & 3 & 6 & 2 & 1 & 2 & 3 & 4 & 3 & 1 & 2 & 1 & 3 & 2 \\
\hline $\mathrm{T}$ & 204 & 99 & 97 & 219 & 52 & 180 & 190 & 129 & 177 & 79 & 202 & 197 & 129 & 202 & 151 & 85 & 215 & 59 & 104 & 196 & 135 & 280 & 186 & 89 & 129 & 226 & 156 & 140 & 150 & 125 \\
\hline D & 0.59 & 0.63 & 0.41 & 0.44 & 0.74 & 0.66 & 0.66 & 0.62 & 0.41 & 0.5 & 0.4 & 0.61 & 0.97 & 0.27 & 0.51 & 0.73 & 0.43 & 0.38 & 0.3 & 0.59 & 1 & 0.81 & 0.77 & 0.59 & 0.5 & 1 & 0.52 & 1 & 0.38 & 0.56 \\
\hline C & 100 & 90 & 96 & 100 & 54 & 100 & 100 & 90 & 100 & 90 & 100 & 100 & 100 & 100 & 96 & 100 & 100 & 74 & 98 & 100 & 100 & 100 & 100 & 80 & 98 & 100 & 100 & 100 & 100 & 96 \\
\hline \multicolumn{31}{|c|}{ MEDIO MUNDO } \\
\hline $\mathrm{X}$ & 3 & 2 & 3 & 2 & 2 & 4 & 1 & 2 & 4 & 2 & 3 & 2 & 1 & 3 & 2 & 1 & 2 & 1 & 2 & 2 & 2 & 1 & 3 & 2 & 2 & 1 & 3 & 2 & 3 & 1 \\
\hline $\mathrm{T}$ & 92 & 191 & 110 & 126 & 200 & 167 & 66 & 146 & 98 & 104 & 136 & 181 & 82 & 196 & 161 & 113 & 133 & 162 & 130 & 113 & 183 & 187 & 83 & 115 & 189 & 42 & 120 & 26 & 354 & 85 \\
\hline $\mathrm{D}$ & 0.8 & 0.51 & 0.49 & 0.58 & 0.63 & 0.47 & 1 & 0.64 & 0.54 & 0.86 & 0.51 & 0.56 & 1 & 0.65 & 0.74 & 1 & 0.56 & 1 & 0.7 & 0.59 & 0.56 & 1 & 0.82 & 0.52 & 0.5 & 1 & 0.77 & 0.5 & 0.36 & 1 \\
\hline C & 70 & 100 & 90 & 98 & 90 & 100 & 86 & 100 & 80 & 64 & 94 & 100 & 90 & 100 & 100 & 100 & 100 & 100 & 90 & 100 & 100 & 100 & 70 & 100 & 100 & 58 & 100 & 100 & 100 & 100 \\
\hline
\end{tabular}

\footnotetext{
${ }^{1}$ Universidad Nacional Mayor de San Marcos - Museo de Historia Natural, Laboratorio de Florística. Av. Arenales 1256, Lima 14 - Perú. haponteu@yahoo.fr

${ }^{2}$ Universidad Nacional Mayor de San Marcos - Museo de Historia Natural, Laboratorio de Florística. Av. Arenales 1256 , Lima 14 - Perú
} 